\title{
METABOLIC FINGERPRINTING IN BALNEOTHERAPY
}

\author{
Munteanu, Constantin ${ }^{1,2, c}$; Munteanu, Diana ${ }^{1,2}$ \\ ${ }^{1}$ Romanian Association of Balneology, Romania \\ ${ }^{2}$ National Institute of Rehabilitation, Physical Medicine and Balneoclimatology, Romania \\ cEmail: secretar@bioclima.ro
}

\begin{abstract}
Research in balneology must be connected to diagnostic tools and paraclinical evaluation of biological status of an organism treated by balneotherapy for various diseases. Using a natural therapeutic factor to treat a certain medical problem has to be substantiated by positive changes in biological parameters of the organism that stand as arguments for the therapeutic property of that natural therapeutic factor. Looking to the results of a study, which evaluates paraclinical parameters as terms for a mathematical matrix, and interpreting the global picture as a mathematic function helps to be objective in recommending a natural therapeutic factor in a specific pathology. Within the system biology framework, using an array of paraclinical parameters changes to evaluate the biological status is of great importance in our research regarding the role in our health status of natural mineral waters. For disease diagnostic and monitoring purposes, using a tool system that gives probabilistic outcomes and quantitative results that could be readily interpreted in biological terms represent an ideally methodical system to argue the effects of mineral water ingestion (Ellis et all, 2007). Experiments can be performed on samples from laboratory animals of the species Wistar rats bred under standard conditions, the cage groups were in adequate numbers so as not to disturb the observation of each animal, temperature $21-22 \mathrm{oC}$, humidity: minimum $30 \%-$ maximum $70 \%$, delighting, conventional diet.
\end{abstract}

KEYWORDS: metabolic fingerprinting, natural therapeutic factor

\section{Introduction}

Metabolic fingerprinting is a strategy to determine the biological status of biological samples using advanced methods for determining the concentration of metabolic products.

Metabolic Fingerprinting provides an array whose data, through mathematical analysis and biological interpretation permit to classify and characterize the biological sample and the extrapolation to the state of the cell or the organism from which the sample was taken and analyzed.

Metabolism is more sensitive to disruption than transcriptomic and proteomic level. Explanation: The activity of various metabolic pathways in the cell body and reflected more accurately in concentration than the concentration of metabolites or enzymes encoded mRNA.
The use of metabolic fingerprinting:

- for microbiological screening mutant collections of microorganisms to identify major alterations in the metabolic pathways;

- analysis of the action of drugs and natural therapeutic factors;

- identification of biomarkers or biological indicators to express the state of the body;

- evaluation of therapeutic effects of drugs / therapeutical natural factors;

- diagnosis.

Knowledge of general issues regarding the effects of natural factors, location and their mechanism of action is fundamental for rational therapy. 
The biological activity of natural factors requires an initial interaction of chemical or physico-chemical order with components of living matter molecules.

Data analysis is the final step in a randomized therapeutic trial because established if the medication was effective, how large was the treatment effect given and how precise was the treatment effect estimate (confidence interval).

The size of the combination is measured as in any cohort study by the relative risk, in contrast to etiological studies, the relative risk is usually subunit, because the treatment is a protective factor, and not one of risk.

\section{Blood tests}

\section{A. HEMATOLOGY}

The hemogram is a basic screening test as one of the most commonly requested laboratory tests, often representing the first step in the establishment the hematological status and diagnosis of various non-hematologic and hematologic disorders.

Hemogram consists of measurement of the following parameters:

- $\quad$ number of leukocytes;

- $\quad$ number of erythrocytes;

- hemoglobin concentration;

- hematocrit;

- the red cell indices: mean corpuscular volume (MCV), mean erythrocyte hemoglobin $(\mathrm{MCH})$, mean hemoglobin concentration (MCHC) and red cell distribution width (RDW);

- $\quad$ the platelet counts and platelet indices: average platelet volume (VTM) and platelet distribution width (PDW)

- $\quad$ differential white blood count;

The erythrocyte number represents the basis for the assessment of erythropoiesis. The erythrocytes are further investigated by measuring the concentration of hemoglobin and hematocrit, and based on their the analyzer will calculate the erythrocyte indices: $\mathrm{MCV}, \mathrm{MCH}$, $\mathrm{MCHC}$ and RDW, which qualitatively characterizes the erythrocyte population.

The erythrocytes are the most numerous blood cells are enucleate and necessary for the tissue respiration. The erythrocytes are the most specialized cells of the body, the main function consisting of transport $\mathrm{O}_{2}$ from lung to tissues and transfer of $\mathrm{CO}_{2}$ from the tissues to the lungs. This is achieved through hemoglobin contained in red blood cells. The biconcave disc shape of the erythrocytes gives the ratio volume / surface of optimal gas exchange and provide them deformability during the microcirculation crossing.

Indications - in combination with hematocrit and hemoglobin concentration, erythrocyte number is useful in the detection and monitoring of anemia and erythrocytosis / polycythemia.

Reference values - different values according to age and sex: 3.8 to $4.8 * 10^{\wedge} 6 / \mathrm{mm} 3$

The hemoglobin. The hemoglobin represents the main component of erythrocytes (95\% of erythrocyte cytoplasmic proteins) and serves as a vehicle for the transport of $\mathrm{O}_{2}$ and $\mathrm{CO}_{2}$.

Hemoglobin is a conjugated protein consisting of a tetramer composed of 2 pairs of polypeptide chains (globin), each of them being conjugated to a heme group, a complex of an iron ion with the red pigment, porphyrin, which gives blood red. Each gram of hemoglobin can carry $1.34 \mathrm{~mL} \mathrm{O}_{2}$ per $100 \mathrm{~mL}$ of blood.

The hemoglobin also serves as a buffer in the extracellular fluid. In tissues at low $\mathrm{pH}, \mathrm{O}_{2}$ dissociates of $\mathrm{Hb}$; deoxygenated hemoglobin binds hydrogen ions, in erythrocyte carbonic anhydrase converts $\mathrm{CO}_{2}$ into bicarbonate and hydrogen ions. As the hydrogen ions bind to hemoglobin, bicarbonate ions leave the cell; for each bicarbonate ion leaving the cell enters a chloride ion.

The forms of hemoglobin normally present in circulation include deoxyhemoglobin (HHB), oxyhemoglobin $\left(\mathrm{O}_{2} \mathrm{Hb}\right)$, carboxyhemoglobin $(\mathrm{COHb})$ and methemoglobin $(\mathrm{MetHb})$, all of which are determined in whole blood together. In certain clinical situations, different forms of $\mathrm{Hb}$ can be individually determined.

The Leukocytes. Leukocytes are divided into two main groups: granulocytes and a-/nongranulocytes. The granulocytes are so named because of the presence in the cytoplasm of different grain sizes and identifies three types of granulocytes by affinity staining the blood smears colored Wright: neutrophils, eosinophils and basophils. In addition, these cells are also called polymorphonuclear leukocytes due to multilobulated nucleus. Nongranulocytes consisting of lymphocytes and monocytes generally do not contain distinct cytoplasmic granulation were non-lobulated nucleus, is also called mononuclear leukocytes.

Reference values - for adults $=4000$ $10000 / \mu \mathrm{L}$ or $4-10 \times 109 / \mathrm{L}$ 


\section{B. BIOCHEMISTRY}

\section{Glucose (serum glucose)}

Glucose is the most significant monosaccharide from the blood which supports cell activity and resulting from the digestion of carbohydrates and the conversion of liver glycogen into glucose. Blood glucose concentration is controlled by the two antagonists hormones insulin and glucagon. Hyperglycemia favors the formation of cytokines and activates the endothelium inflammation, also produces an increase of oxidative stress.

Reference values: 74-106 mg/dl

Decreases in insulinoma, extrapancreatic tumors (e.g. hepatoma), Addison's disease, hypopituitarism, malabsorption and so on.

Increases in diabetes, Cushing's disease, acromegaly / gigantism, pheochromocytoma, adenoma pituitary (growth releasing hormones) and so on.

\section{Serum creatinine}

The creatine is synthesized in the liver and is taken after release is taken up in approximately 98\% from the muscles where takes place phosphorylation, in this form having an important role in muscle energy storage. Creatinine is the fixed nitrogen constituents of the blood, unaffected by most foods, of effort, circadian rhythms and other biological constants and is correlated with muscle metabolism. The main utility of serum creatinine determination is to diagnose the renal failure. Serum creatinine is a more specific and sensitive indicator of renal function than urea. However, chronic kidney disease is useful to determine both creatinine and serum urea.

Reference values: $<1,2 \mathrm{mg} / \mathrm{dl}$

Decreases in decrease in muscle mass, protein deficiency in the diet, severe liver disease.

Increases in acute or chronic kidney disease of glomerular or tubulo-interstitial cause, urinary tract obstruction (postrenal azotemia) and so on.

\section{Serum uric acid}

Uric acid results from degradation of nucleic acids representing the final product of purine metabolism. The main transformation occurs in the liver, where it is transported through the plasma to the kidneys where it is filtered and excreted at a rate of about $75 \%$. The remaining uric acid is removed and degraded in the gastrointestinal tract. Overproduction of uric acid occurs in the following situations: excessive catabolism of nucleic acid (gout), production and massive destruction of cells (leukemia) or inability to excrete the final product (kidney failure).

\section{Reference values: $3,5-6 \mathrm{mg} / \mathrm{dl}$}

Decreases in administration of drugs: ACTH, uricosuric (cortisone, allopurinol), various causes: Wilson's disease, Fanconi syndrome, SIADH, acromegaly (some patients) and so on.

Increases in kidney failure, gout, asymptomatic hyperuricemia (gout should be evaluated periodically) increasing of the destruction of nucleoprotein: leukemia and chronic myeloproliferative syndromes, multiple myeloma, lymphomas (especially irradiation) and so on.

\section{Urea}

The urea is the major final product of nitrogen metabolism of amino acids, derived from cleavage of proteins in the stomach and intestine under the action of proteolytic ferments and their absorption through the intestinal wall. The principal place of urea formation is the liver. The high level of urea in the blood indicates the significant alterations of the glomerular filtration rate. A low value of urea 6-8 mg / dL, is often associated with a state of hyperhydration, a value of $10-20 \mathrm{mg} / \mathrm{dL}$ indicate a normal glomerular function, the blood urea increased to $50-150 \mathrm{mg}$ / $\mathrm{dL}$ requires a significant damage of renal function.

Reference values: $<50 \mathrm{mg} / \mathrm{dl}$

Decreases in severe liver disease (hepatic insufficiency) toxicity, infection, acromegaly, malnutrition, malabsorption and so on.

Increases in kidney disease, acute or chronic (renal azotemia) glomerulonephritis, pyelonephritis, urinary tract obstruction (postrenal azotemia), protein catabolism intensifying (serum creatinine values remain unchanged): burns, cancer, prolonged fever and so on.

\section{ALT)}

Alanine aminotransferase (SGPT / ALT /

ALT (ALAT), alanine aminotransferase or glutampiruvic transaminase (TGP) is an enzyme that belongs to the class transferases and catalyze the reversible transfer of the amino group (NH2) from an amino acid (alanine) $\alpha$ cetoglutarate leading to the formation of pyruvic acid and glutamate. The ALT determination is indicated in the diagnosis and monitoring of acute liver disease.

Reference values: $<55 \mathrm{mg} / \mathrm{dl}$

Decreases in: urinary infections, malignancies, pyridoxal phosphate deficiency (malnutrition, alcohol). 
Increases in: liver metastases, obstructive jaundice, increases may be small and delayed in fatty liver - increases of 2-3 times normal, alcoholic hepatitis $(<150 \mathrm{U} / 1)$, shock, severe burns, infectious mononucleosis.

\section{Cholesterol}

Cholesterol is a component of cell and organoid membranes and a precursor of bile acids, vitamin $\mathrm{D}$ and steroid hormones. Cholesterol is insoluble molecule that moving associated with the lipoprotein HDL, LDL, and VLDL. Quantification of total cholesterol ascertaining the hypercholesterolemia, isolated or associated with hypertriglyceridemia. The determination of the cholesterol level assesses the lipidic status and metabolic disorders, the risk of atherosclerosis, coronary stenosis and myocardial infarction. The normal values vary according to sex, age, diet and so on. Cholesterol is a well known and demonstrated risk factor for cardiovascular and cerebrovascular disease the lipid profile modifications being a mark of the metabolic syndrome.

The relationship between increased levels of plasma cholesterol and atherosclerotic vascular disease fulfills all criteria for causality. The evidence for lowering plasma cholesterol that reduces the risk are unequivocal. The risk is high, and the greater the benefit. A $10 \%$ reduction in total plasma cholesterol is followed by a $25 \%$ reduction in the incidence of coronary artery disease after 5 years, and a reduction in LDL cholesterol of $1 \mathrm{mmol} / 1(\sim 40 \mathrm{mg} / \mathrm{dl})$ is accompanied by a reduction of $20 \%$ of coronary events [194].

Reference values: $<200 \mathrm{mg} / \mathrm{dl}$

Decreases in hipo-/a-beta-lipoproteinemia, Tangier disease, severe hepatocellular damage, hyperthyroidism, myeloproliferative disease, steatorrhea with malabsorption and so on.

Increases in hyperlipoproteinemia type IIb, III, V, type IIa familial hypercholesterolemia, biliary obstruction: cholestasis, biliary cirrhosis, nephrosis, pancreatic disease, pancreatic and prostate cancer, hypothyroidism and so on.

\section{cholesterol \\ HDL (High Density Lipoprotein)}

HDL-cholesterol (HDL-C) is a group of lipoproteins synthesized and secreted by hepatocytes. HDL has an important role in cholesterol metabolism, participating in its transport from extrahepatic tissues to the liver for catabolism and excretion.

\section{Reference values: $40-60 \mathrm{mg} / \mathrm{dl}$}

Decreases in familial hypertriglyceridemia, hepatocellular cholestasis diseases, chronic renal diseases and so on.

Increases in hyper-alfalipoproteinemia (excess HDL) family (autosomal dominant) / secondary (alcoholism, pesticides, estrogen), hypo-beta-lipoproteinemia and so on.

LDL (Low Density Lipoprotein) cholesterol

The low-density lipoprotein that transports approximately $60 \%$ of plasma cholesterol and facilitate cholesterol deposition in peripheral tissues increased the risk of heart and vascular atherosclerosis. LDL cholesterol is responsible for the initiation and maintenance of inflammation in the intima of blood vessels.

\section{Reference values: $50-170 \mathrm{mg} / \mathrm{dl}$}

Decreases in hypolipoproteinemia family, malabsorption, severe burns, malnutrition, hyperthyroidism and so on.

Increases in familial lipoproteinemia, familial hypercholesterolemia, nephrotic syndrome, chronic liver disease, multiple myeloma and so on.

\section{Triglycerides}

The triglycerides represent $95 \%$ of fat reserves and the primary role of providing energy to the cells. Increased serum triglycerides values report the need to seek other factors that may be associated with the so-called metabolic syndrome.

Metabolic syndrome describes the association of cardiovascular risk factors in people with obesity or insulin resistance. Identify individuals with high risk of developing CVD, consistent with associated risk factors, but does not indicate a higher risk of CVD than the effect of the involved risk factors [194].

Reference values: $<150 \mathrm{mg} / \mathrm{dl}$

Decreases in abetalipoproteinemia, malnutrition, malabsorption, hyperthyroidism and so on.

Increases in genetic hyperlipidemia (types I, II-b, III, IV, V apo C-II deficiency) and secondary gout, pancreatitis, liver disease and so on.

\section{Alkaline phosphatase}

Alkaline phosphatase (ALP) is an enzyme found in the liver and bones and is excreted in bile. Is part of hydrolases class and consists mainly of three isoenzymatic forms (hepatobiliary, bone, intestinal), plus a transient form during pregnancy (placental form). The determination of the alkaline phosphatase is 
usually used for differential diagnosis of liver diseases. Another area of clinical uses includes bone disease, being at this time the only enzyme with practical importance for bone pathology, and hyperparathyroidism. In the tumors of various etiologies, alkaline phosphatase has an amount of tumor marker (detection of liver metastases or bone).

Reference values -are dependent on age and sex: 100-290 IU / L

Decreases in hypophosphatase familial, hypothyroidism, cretinism, malnutrition, zinc deficiency, magnesium deficiency and so on.

Increases in hepatic origin most marked increases are found in mechanical obstruction of extrahepatic bile ducts or intrahepatic cholestasis, bone origin: hyperparathyroidism, hyperthyroidism, Paget's disease of bone metastasis, intestinal origin: in various ulcerative diseases of the gastrointestinal tract and so on.

\section{Total proteins}

Plasma contains more than 300 different proteins: enzymes, enzyme inhibitors, clotting factors, antibodies, binding proteins. Except immunoglobulins and hormones, most plasma protein is synthesized in hepatocytes and released into the bloodstream. Hyperproteinemia can be caused by dehydration or may be the result of concentration increasing of specific proteins (immunoglobulins in chronic infections, multiple myeloma and so on). Hypoproteinemia can be caused by hemodilution or excessive loss of protein in chronic kidney disease or severe burns and so on.

Reference values: 6,4-8,3 mg/dl

Decreases in insufficient intake (starvation or malabsorption), liver cirrhosis and chronic alcoholism, glomerulonephritis and nephrotic syndromes and so on.

Increases in dehydration and hemoconcentration due to loss of fluids, some cases of chronic liver disease and other conditions associated with chronic infections / inflammation and so on.

\section{Lactate dehydrogenase (LDH)}

$\mathrm{LDH}$ is an intracellular enzyme widely distributed in the body being encountered mainly in the kidneys, myocardium, skeletal muscle, brain, liver and lungs. Although the LDH increased are nonspecific, this test is useful to confirm the diagnosis of myocardial infarction or lung. In the myocardial infarction, the persistence of increased LDH level is longer than the other enzymes.

Reference values: 150-450 U/1
Increases in in myocardial infarction occur increased LDH levels at 36-55 hours since the debut persisting 3-10 days, LDH increased pulmonary infarction is registered within 24 hours of onset of chest pain and other conditions associated with increased LDH levels: congestive heart failure, liver disease (cirrhosis, alcoholism, acute hepatitis), solid tumors, leukemias, lymphomas, hypothyroidism, muscle diseases (dystrophies, trauma) and so on.

The markers of pro-inflammation. It is known that high levels of IL-6, TNF-alpha, and $\mathrm{C}$-reactive protein are associated with high vascular risk. C-reactive protein is a risk factor for acute cardiac events. Interleukin-6 and chemotactic macrophage protein (macrophage chemotactic protein MCP) are two other markers of inflammation with high value in metabolic syndrome. MCP macrophage chemotactic protein - causes the monocyte migration to inflammation place in the endothelial wall.

\section{IONOGRAM}

Mineral analysis (ionogram) from the blood or urine. Ionogram values vary with the mode of nutrition, age, the season and the type of condition.

The excess or deficiency of metal ions in the body explains more chemical or pathological conditions, while the intermediate concentrations are crucial for normal development. In fact, every body requires an optimum concentration of a given element, over or under which no longer develop properly.

\section{Sodium - sodium levels}

Sodium is a trace mineral with the ability to maintain a constant water balance outside the cells with role in maintaining acid-base balance. Sodium promotes muscular excitability.

Sodium contributes to the proper functioning of the nervous system and muscles. The existing sodium in the body is regulated by the kidneys, which increases or decreases excretion, depending on the amount ingested. The amount of sodium in the body is $80-100$ grams.

Reference values: $310-345 \mathrm{mg} / 100 \mathrm{~mL}$

Decreases abnormal (hyponatremia): insufficient salt intake, dehydration global low fluid ingestion, polyuria (diabetes) chronic renal failure, adrenal insufficiency and so on.

Abnormal growths (hypernatremia): loss of fluid in the extrarenal (excessive sweating, diarrhea, vomiting), loss of fluids by the kidneys (insipid diabetes, kidney failure), heart failure, brain injury, aldosteronism and so on. 


\section{Calcium-Calcium levels}

The calcium is the mineral present in the highest amount in the body (one $\mathrm{kg}$ - three pounds). Almost the entire amount of calcium in the body is fixed in bones and teeth and the rest is distributed in tissues and body fluids. Thus per $100 \mathrm{~g}$ of muscle tissue contains $70 \mathrm{~g}$ of calcium in nerve $15 \mathrm{~g}, 4,5 \mathrm{~g}$ in cerebrospinal fluid and in plasma between 9 and $10 \mathrm{mg}$ calcium.

Calcium concentration in the blood is maintained between $9-11 \mathrm{mg} \%$. Calcium is an essential mineral for maintaining human health. There is only one component of bones and teeth, but is also essential for normal blood clotting and is required for normal functioning of muscles and nerves. The values are generally higher among men than in women with a slight tendency to decrease with age.

The main function of calcium in the body is that with phosphorus to strengthen and maintain in good condition the skeleton and teeth. This is accomplished in optimal conditions when the ratio between the amount of calcium and phosphorus is 2-1. Also calcium can not be absorbed only in the presence of sufficient amounts of vitamin $\mathrm{D}$.

Normal values: 8.1 - $10.4 \mathrm{mg} / 1$ / 4,0-6,0 mg / dL

\section{Potassium}

Potassium is an electrolyte, a molecule positively charged, which functions with other electrolytes such as sodium chloride, sodium and carbon dioxide $\left(\mathrm{CO}_{2}\right)$. It has a role in the proper functioning of both the skeletal muscle and heart. Therefore, lack of potassium from those muscles cause a weakening in their activity. Normally, the excess of potassium from the body due to feeding is eliminated automatically in urine through the kidneys. However in diseases that prevent the excretion of potassium through the kidneys (renal failure, diabetic coma, Addison's disease), it accumulates in the body causing heart problems.

Potassium help to settle the amount of fluid in the body, stimulates muscle contraction and maintain a stable acid-base balance in the body. It is present in all body fluids, but the greatest amount is found in cells.

Since the concentration of potassium in the blood is very small, minor changes may have relevant consequences.

If the potassium levels increase or decrease too much, the patient's health may be threatened: occurs respiratory failure, heart rhythm disorders. An abnormal concentration can affect neuromuscular tissue functions, e. g. heart muscle may lose the ability to contract.

Analysis of Potassium is performed along with other routine medical examinations. The test is used to detect concentrations that are too high (hyperkalaemia) or too low (hypokalaemia). The most frequent cause of hyperkalaemia is kidney disease, but the administration of many drugs can decrease the potassium excretion from the body.

Symptoms of hypokalaemia can occur if there is vomiting and diarrhea or the excess of sweating. Potassium can be lost through urine; in rare cases the potassium level may be low because it does not accumulate enough in the diet. Potassium concentration can be measured at regular intervals to monitor drugs that may lead to a situation in which the kidneys lose potassium, of diuretics.

As well as calcium has a big role in the proper functioning of both the skeletal muscle and heart. Therefore, lack of potassium from those muscles cause a weakening in their activity. Potassium is found in almost all foods but especially in fruits (apples, cherries, pears) and vegetables.

Normally, the excess of potassium from the body due to alimentation is automatically eliminated through the kidneys in urine. In diseases in that are preventing the excretion of potassium through the kidneys (renal failure, diabetic coma, Addison's disease), it accumulates in the body causing heart problems.

Potassium decreases in the blood when consuming foods low in this mineral as white bread, refined sweets or by diuretics based on cortisone. Also, laxative and purgative medicines eliminated with the feces and large amounts of potassium. Therefore, when the potassium is analyzed, will be interrupt these medications with 48 hours before the analysis. Sweating, vomiting and diarrhea, especially in young children, diabetes, prolonged fasting are common causes of loss of potassium from the body.

Normal values: $3.5-4.5 \mathrm{mEq} / \mathrm{L}$

\section{Iron - Iron levels}

Through this test can determine the amount of iron in the body (percentage concentration of iron in the plasma), which has different values according to age.

The iron content of the body in the circulating blood are divided into two sectors: in erythrocytes in hemoglobin structure (about $3 \mathrm{~g}$ ) in plasma (about $3 \mathrm{mg}$ ). 
It is the main mineral in the composition of red blood cells, giving it its intense red color. Also, the iron take part in the formation of enzymes critical to the body, such as enzymes which contribute to determining the oxygen in the cell. The iron from the body comes from water and especially iron-rich foods (meat, liver, spleen, eggs, carrots, spinach, soy, chocolate and others).

The body needs of iron is low, $2 \mathrm{mg} /$ day, so the 10 to $15 \mathrm{mg}$ per day which man receives from food and water consumed are enough for the body. However, in adults and especially for children malnourished, lack of iron in the diet can lead to anemia spared.

The decrease of iron in the body is found after large hemorrhage or repeated and low bleeding even after as bleeding from the nose, in the urinary and the genital, from the digestive tract (hemorrhoids) and so on. The digestive diseases, surgery on the stomach and intestine produce anemia that reduce iron absorption in the body. Anemia through iron deficiency is determined only by the physician after blood analysis.

\section{Normal levels of iron:}

Mens $\quad 0,90-1,50 \quad \mathrm{mg} / 1 \quad(90-150 \quad$ ug or gamma/100 ml)

Female $=0,8-1,40 \mathrm{mg} / \mathrm{l}(80-140 \mathrm{ug}$ or gamma/100 ml).

\section{Magnesium - Magnesium levels}

Magnesium is a mineral that is found in every cell in the body being vital for energy production, muscle contraction, nervous system function and bone system.

This test measures the amount of magnesium in the blood whose is present in only a small percentage (about 1\%) of the total volume.

Approximately half of the total amount of magnesium is combined with calcium and phosphorous to help bone formation. The magnesium is found in a wide variety of foods such as green vegetables (spinach) and other nutrient sources.

$\mathrm{Mg}^{2+}$ has an important role in glycolysis, cellular respiration, transmembrane calcium transport. In the muscle cells, $\mathrm{Mg}^{2+}$ acts as an antagonist of calcium.

$\mathrm{Mg}^{2+}$ activates $\mathrm{Na}-\mathrm{K}$ ATPase, and therefore, plays a role in cardiac arrhythmia.

$\mathrm{Mg}^{2+}$ plays a role in reducing muscle contraction and vascular tone inhibiting intracellular calcium availability in myocardial cells and vascular myocytes. Naturally $\mathrm{Mg}^{2+}$ is a blocker of $\mathrm{Ca}^{2+}$.

$\mathrm{Mg}^{2+}$ homeostasis is regulated by intestinal absorption (small intestine) but especially by renal secretion. It is a chemical element indispensable for proper functioning of the human body organs: liver, muscle, nervous system and so on. It composes the enzymes taking part in the burning of glucose in the body and interferes with calcium in muscle activity.

Increasing of the blood concentration of magnesium is found in renal insufficiency (because the kidneys can not eliminate it in the urine), in severe diabetes, in Basedow disease (thyroid hyperfunction).

Decrease in blood magnesium levels in the thyroid insufficiency after excessive losses through vomiting and diarrhea or urine after diuretic and in chronic alcoholism.

Lack of magnesium is felt more to children than to adults.

Normal magnesium levels: - 1.6-2.55 mg / dl The pathological decreases Hypomagnesaemia

Clinical symptoms are similar to those of hypocalcemia and $\mathrm{Mg}$ homeostasis is often deregulated simultaneously with that of $\mathrm{Ca} . \mathrm{Mg}$ decreases through loss in kidney (clinical signs occur in $\mathrm{Mg}$ values $<1.22 \mathrm{mg} / \mathrm{dl}$ ) due to: nephrotoxic drugs cisplatin, aminoglycosides, methotrexate, diuresis (furosemide, thiazide), alcoholism, intestinal malabsorption, endocrine disorders aldosteronism, hyperparathyroidism, hyperthyroidism, diabetic ketoacidosis associated with familial hypomagnesemia with hypercalciuria and hypermagneziuria, long-term use of diuretics, prolonged diarrhea, postoperative status, severe burns, gastrointestinal disturbances, intoxication and so on.

The pathological increases
hypermagnesaemia

An increased level of magnesium is very rare due to dietary sources, being the result of excretion problems or excessive supplementation. Clinical symptoms occur at levels of $6.08 \mathrm{mg}$. / Dl and the values of 12, 2 $\mathrm{mg}$. / Dl and appears to paralyze the respiratory muscles, acute and chronic kidney disease, excessive intake of antacids or $\mathrm{Mg}$ preparations, hypothyroidism, dehydration, diabetic acidosis.

\section{Urine analysis}

Biochemistry. The urine is a biological fluid of excretion, with a complex chemical composition, which can vary in certain pathological states. Full analysis of urine include 
determining the physical characteristics (color, appearance, specific gravity), chemical $(\mathrm{pH}$, protein, glucose, ketones, blood cells, bilirubin, urobilinogen, leukocytes, nitrite) and microscopic examination of sediment.

In the context of the complex picture of early diagnosis, treatment and prevention of diseases associated with age, picture containing many unquantifiable and independent variables, difficult to analyze, appears to be necessary the analysis, mathematical modeling and simulation of bio-medical relations of laboratory parameters. The mathematical model is an isomorphic representation of the reality that facilitates the discovery of the analyzed parameter links and thus allows the evaluation of evolution of the biological status of the body, respectively the rate of aging. "Health status" implies the homeostasis of the system represented by the body.

Multicompartment models utilised in mathematical modeling are used in many areas such as pharmacokinetics, epidemiology, biomedicine, systems theory, electrical circuits, complexity theory, engineering, physics, information systems, etc.

Using compartmental modeling systems is based on a number of assumption and approximations that are used in all applications that utilize this type of modeling:

$>$ inside a compartment each time point energy or materials are distributed homogeneously;

$>$ the material or energy exchange between compartments depends on the density of these compartments;

$>$ commonly, it is desirable that the materials passing through the compartments do not react chemically during the transfer;

$>$ If in the pattern occurs the cell concentration, usually is required a constant volume for that compartment, which in reality is not completely true.

Thus, for example, the mathematical model of diabetes, which will be used in an adapted version of the aging process analysis, includes 19 differential equations and 47 parameters, which leads to the use of specialized software to solve such systems. In the last years, the analysis of intravenous glucose tolerance test and insulin levels test continue to be widely used in studies of susceptibility and resistance to insulin.

To ensure collection of sufficient quantity and quality data for subsequent statistical analyses is important to avoid "information rich
- poor information" syndrome related to data collection [9].

Before proceeding to the statistical analysis, the data obtained should be prepared tu use standard methods of summarizing, presenting and checking for identification of "aberrant" results. If not detected in time these values can have profound effects on subsequent statistical analyses. Basic graphical presentations of data are useful for highlighting differences, for example: a profile can show the changes in the concentrations of some metabolites. Before being subjected to comprehensive analysis, the data must undergo a preliminary examination to verify their integrity. Also, must be taken into account missing data or those that are below detection limits [9].

One of the most significant challenges for data analysis phase is to extract a "signal" of an inherent "noisy" environment [9].

Most statistical methods are based on elements of classical statistical analysis (analysis of variance, $t$ tests, $F$ tests, and so on). These methods are used by researchers from various fields and disciplines. However, when analyzing biological processes, it is difficult to justify the assumption that the response variables are normally distributed that the variance is constant in space and time and that the observations are not correlated. In these cases, the remedial action (data processing) overcomes some of the difficulties, but it is more likely that a statistical alternative is required. For example, generalized linear models are often more suitable than classical techniques for data analysis ANOVA, due to their recognition and inherent treatment as variables with abnormal response [9].

On the market, there are data analysis software programs that are worth mentioning: SAS ${ }^{\circledR}$ - for the analysis of large data sets, MINITAB ${ }^{\circledR}$, the STATISTICA ${ }^{\circledR}$, SYSTAT $\circledR$, S-PLUS ${ }^{\circledR}$ that are used to analyze datasets of medium size. Their popularity is due to their ease of use. All these software programs provide a high level of functionality and technical sophistication, but should not be abused in their application [9].

The data obtained in the laboratory should be summarized in a form which permits their analysis. It is good that data to be checked and to be accepted only those that comply with quality criteria (required by QA / QC analysis) [9].

Analytical data can be registered in spreadsheets that are then used for analysis. Physical measurements should be registered in tables with a format allowing quick comparison 
with chemical and biological information for the same parameters.

Data integrity. Disruption of the integrity of data quality (losses or errors) can occur in several ways: during the preparation of samples for analysis, either during registration, handling or electronic processing [9].

The concentration of data is the first significant step in data processing and allow presentation and summarizing of significant characteristics because it helps the analyst to identify extreme values or aberrant in certain situations. For data concentration can be used a combination of statistical tools. These are graphs (histograms, lines, boxes, dots and dispersion), tables (frequency distributions), numeric values (the mean, median, standard deviation, percents). The objective to summarize statistical calculations is to transform contained data into a concise and as possible clear form to estimate a parameter of some values populations [9].

Frequency tables are commonly used for summarizing data because they can condense large data sets in a form easily handled without substantial loss of information and that can be presented graphically as histograms and bars [9].

Typically, are used statistics that measure central tendency or "the average", such as the mean, median and mode. For applications that analyze the quality, the arithmetic mean, the geometric mean and median are the most suitable. The median is a robust estimator of central tendency because it is relatively indifferent to extreme dates. The arithmetic mean does not have this property, however, it is used most often because it is easy to calculate and uses all data. It has well-founded statistical properties and many statistical tests based their conclusions on an average population [9].

The choice of the method should not be automatic but will depend on the circumstances of the moment. For arithmetic geometric and harmonic mean, the following statement is always true: the harmonic mean $\leq$ geometric mean $\leq$ arithmetic mean. The three averages are equal if all sample values are identical [9].

Another very important feature is the results distribution is the variability. The simplest measure of variability is the variation domain the difference - between the highest and lowest value. The variation domain is rarely used as a measure of variability because it is strongly affected by outliers in the data set (after all he is defined as the difference between the two extreme values). The measure most often used when variability is concern, is the variance (S2) or its square root, the standard deviation (S). The standard deviation is preferred because it has the same measurement units as the original data [9].

One of the difficulties regarding that the standard deviation is the fact that is not readily comparable for different populations of samples that tend to be numerically higher as a result of a growing average. This difficulty can be overcome by using the coefficient of variation (CV) that is defined as the ratio between the standard deviation and the mean.

Other statistical entity that has proven useful is the percentile of a distribution. The percentile $\boldsymbol{p}$ is a value that is less than or equal to $p \%$ of the total amounts of distribution, e.g. 50\% of all values of the distributions are numerically less than or equal to 50 percentile (known as median) while $80 \%$ of the values are numerically less than or equal to 80 percentile. The 25,50 , and 75 percentiles are called quartiles (noted by Q1, Q2 and Q3) because they divide the distribution into four parts of equal probability [9].

View data. Thanks to advances in "hard" and "soft" domain, the sophisticated and quality graphics have become more accessible. Graphing is recommended for significant data before any processing or statistical analysis (Tufte, 1983) [9].

Simple graphical tools may assist statistical analyses and facilitate the interpretation of data in various situations as data anomalies and errors, abnormalities, property distributions (location, dispersion,inclination), temporal trends, spatial, attributes, relations (presence and type), checking assumptions corresponding to distributions (e.g. normal probability), time series analysis, reducing the number of dimensions (large data visualization through their projection to lower dimensions), operational performances (control graphics) [9].

The transformation of the data. Mathematical transformations of the data are taken to one of the following objectives: to restore a greater degree of linearity between two or more variables, to stabilize the variance of variables in time, space and for other assigned, to restore a greater degree of normality in the distribution of variables [9].

A number of standard statistical procedures (ANOVA, t test) are relatively robust when there are low or moderate deviations from normal values. Rather than try to achieve normality, the analyst should ensure that data 
distribution has a reasonable degree of symmetry. It is more relevant to check whether the data have homogeneous variances (the variances of the variables are constant for different groups, time, space) and are independent. The data are correlated spatially or temporally (or both) are not influenced by statistical tests described [9].

Checking assumptions regarding the distribution. Many statistical methods are based on the assumption that the data samples were randomly selected from a larger population of values that are normally distributed. There are several reasons for which normal distribution enjoys an essential role in statistical and application theory. First, many of the natural normal phenomena present such a distribution. Then, the importance statistical theorem of the central limit assures us that even if the distribution of independent observations is nonnormal, the aggregate quantities (such as the arithmetic mean) tend to have a normal distribution. What is necessary, is our ability to decide whether a particular set of data can come from a population of values whose distribution is normal or other specified type [9].

Detecting trends. One of the main objectives of monitoring programs is ongoing assessment of changes (temporal and spatial). In many cases, it is required by the need to compare the quality of analyzed components with standard values [9].

Trend analyses can be done using statistical methods that can be simple descriptive methods such as time series representations, or more sophisticated methods such as modeling techniques that try to separate a signal from " general noise".

Standardization. Given the wide variability of most of the processes that taking place in the body (or indirect processes that affect them), it is not surprising that data quality has a high spatial and temporal variability. This natural variation frame tends to mask the changing trends of quality parameters and reduces our ability to distinguish the "signal" from the "noise". Simple graphical tools, such as dispersion graphs and time series can only provide a combined image of both elements, trends and noise [9].

Hypothesis testing. Tests for statistical hypothesis are known as parametric or nonparametric tests. The lack of complete information may result in errors.
Exploring correlations - Relationships between pairs of variables can be conveniently evaluated with standard statistical techniques: correlation analysis and regression analysis.

Correlation analysis. It is done by calculating the correlation coefficient (Pearson) which is a numerical measure of the degree of linearity of the two variables $\mathbf{X}$ (independent variable) and $\mathbf{Y}$ (the dependent variable) [9].

The correlation coefficient varies in the range $-1 \leq \mathrm{r} \leq 1$.

The regression analysis. Typically, the goal of regression analysis is to describe the relationship between a single dependent variable (Y) and a set of independent variables (X1, X2, $\ldots, \mathrm{Xn})$. This is the case of multiple regression [9].

Simple linear regression refers to problems involving only a single $\mathrm{Y}$ and a single $\mathrm{X}$. Most often the identification of the independent variable and the dependent variable is evident. In other cases, this is not possible and then the choice is made arbitrarily.

Multidimensional

Scaling

(Multidimensional scaling - MDS) is a statistical technique that seek to reveal relationships between a large number of variables reconstructing the similarities between pairs of variables in a small space. Biologists and ecologists have found that MDS analyses are particularly useful in highlighting the spatial temporal complex interactions between the biological variables [9].

MDS has some limitations, including the presentation of results in an abstract space, the uncertainty regarding the appropriate size of a small space and a lot of similarity measurements based on MDS.

MDS supporting calculations are extremely complex and are iterative, and a final solution is generally found only once explored several alternatives. The lack of correlation between similarities or distances in the final representation of MDS data and original input data is measured by so-called "statistic of stress" [9]. 


\section{REFERENCES}

1. Convertino VA, Armstrong LE, Coyle EF, Mack GW, Sawka MN, Senay LC Jr, Sherman WM - Adequate fluid replacement helps maintain hydration and, promotes the health, safety, and optimal physical performance of individuals participating in regular physical activity. American College of Sports Medicine position stand: Med Sci Sports Exercise 1996 Jan, 28 (1): I-VII.

2. Ellis David I., Dunn W.B., Griffin J.L., Allwood J.W., Goodacre R. - METABOLIC FINGERPRINTING AS A DIAGNOSTIC TOOL - Pharmacogenomics (2007) 8(9), 1243-1266, ISSN 1462-2416, DOI: http://dx.doi.org/10.2217/14622416.8.9.1243

3. Cinteza D., Munteanu C., Poenaru D., Munteanu D., Petrusca I., Dumitrascu D. - THE THERAPEUTIC EFFECT OF CARBOGASEOUS NATURAL MINERAL WATERS IN THE METABOLIC SYNDROME, Balneo Research Journal, Vol.4, No.1, pp. (2013), DOI: http://dx.doi.org/10.12680/balneo.2013.103 $\underline{8}$

4. Costantino M., Giuberti G., Caraglia M., Lombardi A., Misso G., Abbruzzese A., Ciani F., Lampa E. - Possible antioxidant role of SPA therapy with chlorine-sulphur-bicarbonate mineral water, Springer AMINO ACIDS, February 2009, $36 \quad$ (2), 161-165, DOI: http://dx.doi.org/10.1007/s00726-008-

$\underline{0032-y}$
5. Minich D.M., Bland J.S., Acid - Alkaline Balance: Role in chronic disease and detoxification, Alternative Therapies, 2007, 13 (4),

65, http://www.metadocs.com/pdf/publications/0 707 minich.pdf

6. Türkez H., Geyikoğlu F., Colak $\mathrm{S}-$ The protective effect of boric acid on aluminuminduce hepatotoxicity and genotoxicity in rats, Turk J Biol 35(2011) 293-301, DOI: http://dx.doi.org/10.3906/biy-0902-11

7. Burckhardt Peter - The effect of the Alkali Load of Mineral Water on Bone Metabolism: Interventional Studies, The Journal of Nutrition, 138 (2008), 435S-437S

8. Munteanu C., Munteanu Diana - Lithium mineral waters, Balneo Research Journal, Vol.2, No.1, pp.6-9

(2011), DOI: http://dx.doi.org/10.12680/balneo.2 $\underline{010.1010}$

9. Ciolpan Octavian, Monitoringul integrat al sistemelor ecologice, Bucureşti, Eitura Ars Docendi, 2005, ISBN 973-558-191-4 